\title{
An evaluation of gout visits in the United States for the years 2007 to 2011
}

\author{
Kristen E. Castro, Kaitlyn D. Corey, Diana L. Raymond, Michael R. Jiroutek ${ }^{*}$ and Melissa A. Holland
}

\begin{abstract}
Background: This study analyzed visits for and factors associated with gout and gout medication treatment trends for the years 2007-2011 in the United States given the introduction of febuxostat, the first new treatment option for gout in over 40 years, which was introduced to the market in 2009.

Methods: This study was a retrospective, cross-sectional, observational study of patients age 20 and older seen by providers who participated in the National Ambulatory Medical Care Survey (NAMCS), the National Hospital Ambulatory Medical Care Survey Outpatient Department (NHAMCS-OPD) or Emergency Department (NHAMCS-ED) in the United States. The outcome of interest was visits for gout diagnosis and visits where a gout medication was prescribed.
\end{abstract}

Results: Approximately $1.2 \%$ of visits had a diagnosis of gout. There was a significant increase in the percentage of visits with a diagnosis of gout in years 2009-2011 compared to 2007-2008, which remained after adjusting for covariates of interest. Groups more likely to have a visit with gout included those $\geq 65$ and 45-64 (both as compared to those 20-44), the African-American and 'Other' race groups (as compared to Caucasians) and those on a diuretic. Groups less likely to have a visit with gout included females, Hispanic/Latinos, those with insurance type of 'Other' and Medicaid (both as compared to private insurance) and visits to a hospital emergency setting (as compared to physician's office visits).

Conclusion: Although there was a significant increase in visits where gout is diagnosed across study years, the overall percentage of visits with a gout diagnosis is low in the US population. Treatment trends over the study years has remained consistent, with the introduction of febuxostat appearing to have little impact for the study years through 2011.

Keywords: Gout, Febuxostat, NAMCS, NHAMCS-OPD, NHAMCS-ED

\section{Background}

Gout is a type of inflammatory arthritis associated with the formation of urate crystals in the joints. It is estimated that approximately $4 \%$ of Americans are affected, but previous research has shown the prevalence of gout is increasing [1]. Contributing factors to gout include increase in obesity, hypertension, and purine-rich diets [2]. The severity and progression of gout has been directly correlated to an increase in age. [3] Gout is known to be more predominant in males, but is seen in postmenopausal women. The prevalence of gout is higher in African Americans as compared to Caucasians, with

\footnotetext{
* Correspondence: jiroutekm@campbell.edu

Campbell University College of Pharmacy \& Health Sciences, 180 Main Street PO Box 1090, Buies Creek, NC 27506, USA
}

increasing prevalence overall across all demographics [3]. Prior to 2009, pharmacological treatment options for gout had not changed in many years. A new treatment option, febuxostat, was approved by the Food and Drug Administration in February 2009 [4].

No studies have evaluated the proportion of visits with a gout diagnosis since 2009 when febuxostat was introduced to the market. Previous studies have shown that an above normal BMI, hypertension, dyslipidemia, use of a diuretic and older age are associated with an increased risk of gout [5-10].

With the first new gout therapy in over 40 years introduced to the market in 2009, this study sought to evaluate changes in gout-related ambulatory and emergency department visits for the years 2007 through 2011 (the

(C) The Author(s). 2018 Open Access This article is distributed under the terms of the Creative Commons Attribution 4.0 International License (http://creativecommons.org/licenses/by/4.0/), which permits unrestricted use, distribution, and reproduction in any medium, provided you give appropriate credit to the original author(s) and the source, provide a link to the Creative Commons license, and indicate if changes were made. The Creative Commons Public Domain Dedication waiver (http://creativecommons.org/publicdomain/zero/1.0/) applies to the data made available in this article, unless otherwise stated. 
most recent data available) as well as assess factors associated with gout [11]. Additionally, gout treatment trends were plotted to determine the impact of febuxostat on gout therapy since its introduction to the market.

\section{Methods}

This retrospective, cross-sectional, observational study analyzed data collected in the National Ambulatory Medical Care Survey (NAMCS) and the National Hospital Ambulatory Medical Care Survey Outpatient Department \& Emergency Department (NHAMCSOPD/NHAMCS-ED) during the years 2007-2011. Hundreds of reports, manuscripts and books based on data from these widely utilized and respected surveys have been published since the 1970s (https://www.cdc.gov/ nchs/data/ahcd/namcs_nhamcs_publication_list.pdf).

The NHAMCS is an annual, national probability sample of ambulatory visits made to non-federal, general, and short-stay hospitals in the US conducted by the Centers for Disease Control and Prevention, National Center for Health Statistics (NCHS). The multi-staged sampling design is composed of four stages and includes visits to both selected emergency care departments as well as hospital outpatient departments [12-14]. The NAMCS is an annual, national probability sample of visits made to the offices of non-federally employed physicians classified by the American Medical Association or the American Osteopathic Association as "officebased, patient care" (excluding anesthesiologists, pathologists and radiologists) [12-14]. For more information regarding the survey instruments, scope and sample design, data collection and processing, estimation procedures and reliability of survey estimates, go to http:// www.cdc.gov/nchs/ahcd/ahcd_questionnaires.htm.

NAMCS, NHAMCS-OPD and NHAMCS-ED datasets covering five years (2007-2011) were included in this study. Patients 20 or older from any of the three databases were coded as included in the final analysis dataset. There were no exclusions for the study. Across all five years a total of 128,734 raw records in the NHAMCS-OPD, 126,836 raw records in the NHAMCSED and 126,651 in the NAMCS databases met the inclusion criteria (382,221 combined). The study was submitted to the Campbell University Institutional Review Board and received an exemption due to the data sources used being publicly available and de-identified. As such, since this research was based solely on the analysis of previously collected, de-identified data, it complies with the Helsinki Declaration.

The survey data were analyzed using the sampled visit weight that is the product of the corresponding sampling fractions at each stage in the sample design. The sampling weights have been adjusted by NCHS for survey nonresponse as appropriate within each database, yielding unbiased national annual estimates of visit occurrences, percentages, and characteristics [12].

Because of the complex sample design, sampling errors were determined using the SAS SURVEYFREQ and SURVEYLOGISTIC procedures which take into account the clustered nature of the sample [15]. The appropriate NOMCAR and DOMAIN statements/options were implemented in these procedures as recommended by the NCHS [12].

The dependent variable of interest was a diagnosis of gout, where the denominator is the number of visits meeting the inclusion/exclusion criteria. A diagnosis of gout was defined by the appropriate diagnosis codes found in any of the DIAG1-DIAG3 diagnosis fields or appropriate gout medication codes for allopurinol, febuxostat, colchicine, probenecid, and colchicineprobenecid found in any of the DRUGID1-DRUGID8 medication fields.

Rao-Scott chi-square tests were used to analyze whether the proportion of visits with a diagnosis of gout differs by year group (2007-2008 vs. 2009-2011) and whether any association exists between visits with a diagnosis of gout and each of the following variables: age, sex, race, ethnicity, region (US geographic regions included Northeast, Midwest, South, and West), metropolitan statistical area (MSA), insurance status (private, Medicaid or Children's Health Insurance Program [CHIP]/State Children's Health Insurance Program [SCHIP], Medicare, and other [worker's compensation, self-pay, no charge/charity, other], setting type (physician office, hospital outpatient department, hospital emergency department) and diuretic use. These variables were grouped for analysis as shown in Table 1. Odds ratios (ORs), corresponding 95\% confidence intervals (CIs) and $p$-values were reported.

A multivariable logistic regression model was also constructed in order to evaluate the predictive value of all the independent variables of interest simultaneously on visits with a diagnosis of gout, adjusting for covariates of interest. As a primary model filter, only variables with an overall chi-square test of association $p$-value $<0.2$ were included in the multivariable model (year group was included regardless). ORs with corresponding $95 \% \mathrm{CIs}$ and $p$-values for each level of each variable included in the model (in comparison to each variable's reference group) were reported. No collinearity issues between the independent variables included in the model were found. All analyses were generated using SAS software, version 9.3. Plots of the percentage of visits per year with gout medication by drug class and individual drug were constructed to descriptively assess gout treatment trends.

Per NCHS recommendations, any variable with a survey estimate based on either less than 30 records, a 
Table 1 Demographics and Patient Characteristics $(N=382,221)^{a}$

\begin{tabular}{|c|c|}
\hline Characteristic & No. (\%) of Patient Visits \\
\hline \multicolumn{2}{|l|}{ Observation Period } \\
\hline 2009-2011 & $588,791,594(61)$ \\
\hline $2007-2008$ & $375,839,802(39)$ \\
\hline \multicolumn{2}{|l|}{ Age (years) } \\
\hline Mean (SE) & $53.9(0.23)$ \\
\hline \multicolumn{2}{|l|}{ Age Group } \\
\hline$\geq 65$ & $299,032,423(31)$ \\
\hline $45-64$ & $351,209,142(36)$ \\
\hline $20-44$ & $314,389,831$ (33) \\
\hline \multicolumn{2}{|l|}{ Sex } \\
\hline Female & $587,394,025(61)$ \\
\hline Male & $377,237,371(39)$ \\
\hline \multicolumn{2}{|l|}{ Race } \\
\hline Other & $33,516,436(5)$ \\
\hline African-American & $99,604,070(14)$ \\
\hline Caucasian & $602,374,076(82)$ \\
\hline \multicolumn{2}{|l|}{ Ethnicity } \\
\hline Hispanic/Latino & $79,262,491(11)$ \\
\hline Non-Hispanic/Latino & $630,345,157(89)$ \\
\hline \multicolumn{2}{|l|}{ Region } \\
\hline Northeast & $189,690,837(20)$ \\
\hline Midwest & $206,775,296(21)$ \\
\hline West & $201,670,759(21)$ \\
\hline South & $366,494,504(38)$ \\
\hline \multicolumn{2}{|l|}{ MSA } \\
\hline Non-MSA & $120,846,147(13)$ \\
\hline MSA & $843,785,249(87)$ \\
\hline \multicolumn{2}{|l|}{ Insurance Status } \\
\hline Other & $98,736,670(11)$ \\
\hline Medicaid & $88,374,398(10)$ \\
\hline Medicare & $280,902,288(30)$ \\
\hline Private Insurance & $461,730,141$ (49) \\
\hline \multicolumn{2}{|l|}{ Setting Type } \\
\hline Hospital Emergency & $94,712,528(10)$ \\
\hline Hospital Outpatient & $78,137,131(8)$ \\
\hline Physician's Office & $791,781,738(82)$ \\
\hline \multicolumn{2}{|l|}{ Diuretic Use } \\
\hline Yes & $71,243,249(7)$ \\
\hline No & $893,388,147$ (93) \\
\hline \multicolumn{2}{|l|}{ Gout } \\
\hline Yes & $11,769,697(1)$ \\
\hline No & $952,861,700$ (99) \\
\hline
\end{tabular}

MSA Metropolitan Statistical Area

aUnweighted, raw study sample size

${ }^{\mathrm{b}}$ Survey weighting and clusters accounted for reflecting unbiased, national annual estimates of visit occurrences for the portion of the population meeting the study inclusion/exclusion criteria relative standard error of more than $30 \%$, or more than $30 \%$ missing data was excluded from the analyses due to potential unreliability [12]. As a result, the variables of interest weight status, tobacco use, depression, hypertension, and diabetes were excluded from all analyses due to not meeting one or more of the above listed criteria. Missing values were treated as missing in the statistical evaluation. No adjustments for multiple comparisons were made and $p$-values $<0.05$ were considered statistically significant.

\section{Results}

During the study period (2007-2011), the NAMCS, NHAMCS-OPD, and NHAMCS-ED datasets include a total of 495,370 patient visits (unweighted, raw data). A total of 382,221 patient visits within this five-year period met the inclusion criteria and were included in this study (Table 1). Most variables had no missing data, however, ethnicity, race and insurance status were missing 21, 16 and 5\%, respectively. Gout was diagnosed in just over $1.2 \%$ of all patient visits. More patient visits occurred during the more recent half of the study period (61\% in the years 2009-2010). The mean age (SE) was $53.9(0.23)$ years, with a similar percentage of patients in each of the three age groups. Of the patient visits included in the analyses, $61 \%$ were female, $82 \%$ were Caucasian, 14\% were African-American, and 11\% were Hispanic/Latino. Nearly twice as many visits occurred in the South (38\%) as compared to the other regions $(21 \%$ in the Midwest, $21 \%$ in the West and $20 \%$ in the Northeast). However, visits in metropolitan areas represented $87 \%$ of the study total. Private insurance was presented at the majority of all patient visits (50\%), with Medicare presented at $30 \%$ of visits and Medicaid at $10 \%$. The vast majority of visits occurred in a physician's office $(82 \%)$, reflecting the NAMCS survey data (collected in physician offices). Only $7 \%$ of patient visits reported diuretic use.

The primary analysis showed a $30 \%$ relative increase in the percentage of patients who had a visit with a diagnosis of gout in the years 2009-2011 compared to 20072008 (1.3\% vs. $1.0 \%$, respectively; OR 1.28 , 95\% CI 1.10 1.49) (Table 2). The individual chi-square tests of the other covariates of interest that comprised the first part of the secondary analysis, showed a significant association between visits with a diagnosis of gout and the following variables: age, sex, ethnicity, race group, insurance status, setting type, region and diuretic use. See the univariable columns in Table 2 for the details of these individual associations with visits for a diagnosis of gout.

The weighted multivariable logistic regression model, allowing adjustment for the effect of potentially 
Table 2 Gout Predictor Variables, Univariable and Multivariable Analyses* Data are given as number (\%) of patients

\begin{tabular}{|c|c|c|c|c|c|c|}
\hline \multirow[b]{2}{*}{ Parameter } & \multirow[b]{2}{*}{ Gout (\%) } & \multirow[b]{2}{*}{ No Gout (\%) } & \multicolumn{2}{|l|}{ Univariable } & \multicolumn{2}{|l|}{ Multivariable } \\
\hline & & & OR (95\% Cl) & $P$ & OR $(95 \% \mathrm{Cl})$ & $P$ \\
\hline \multicolumn{7}{|l|}{ Observation Period } \\
\hline 2009-2011 & $7,849,421(1.3)$ & $580,942,174(98.7)$ & $1.28(1.10-1.49)$ & 0.0010 & $1.24(1.02-1.52)$ & 0.0346 \\
\hline 2007-2008 & $3,920,276(1.0)$ & $371,919,526$ (99.0) & Referent & - & Referent & - \\
\hline \multicolumn{7}{|l|}{ Age Group (years) } \\
\hline$\geq 65$ & $7,113,034(2.4)$ & $291,919,369$ (97.6) & $8.64(7.04-10.60)$ & $<0.0001$ & $4.94(3.69-6.62)$ & $<0.0001$ \\
\hline $45-64$ & $3,772,290(1.1)$ & $347,436,851$ (98.9) & $3.85(3.11-4.77)$ & $<0.0001$ & $2.52(1.97-3.21)$ & $<0.0001$ \\
\hline $20-44$ & $884,372(0.3)$ & $313,505,459$ (99.7) & Referent & - & Referent & - \\
\hline \multicolumn{7}{|l|}{ Sex } \\
\hline Female & $3,055,558(0.5)$ & $584,338,467$ (99.5) & $0.22(0.20-0.25)$ & $<0.0001$ & $0.20(0.18-0.24)$ & $<0.0001$ \\
\hline Male & $8,714,139(2.3)$ & $368,523,233(97.7)$ & Referent & - & Referent & - \\
\hline \multicolumn{7}{|l|}{ Race } \\
\hline Other & $574,306(1.7)$ & $32,942,130(98.3)$ & $1.43(1.03-1.99)$ & 0.0332 & $2.02(1.47-2.77)$ & $<0.0001$ \\
\hline African-American & $1,226,437(1.2)$ & $98,377,632(98.8)$ & $1.02(0.82-1.27)$ & 0.8513 & $1.33(1.03-1.72)$ & 0.0271 \\
\hline Caucasian & $7,264,368(1.2)$ & $595,109,707$ (98.8) & Referent & - & Referent & - \\
\hline \multicolumn{7}{|l|}{ Ethnicity } \\
\hline Hispanic/Latino & $511,614(0.6)$ & 78,750,878 (99.4) & $0.49(0.36-0.67)$ & $<0.0001$ & $0.64(0.48-0.86)$ & 0.0032 \\
\hline Non-Hispanic/Latino & $8,252,946(1.3)$ & $622,092,211(98.7)$ & Referent & - & Referent & - \\
\hline \multicolumn{7}{|l|}{ Region } \\
\hline Northeast & $2,019,734(1.1)$ & $187,761,102$ (98.9) & $0.90(0.72-1.13)$ & 0.3629 & $0.80(0.62-1.03)$ & 0.0823 \\
\hline Midwest & $3,053,963(1.5)$ & $203,721,333$ (98.5) & $1.25(1.04-1.52)$ & 0.0169 & $1.16(0.95-1.43)$ & 0.1501 \\
\hline West & $2,367,739(1.2)$ & 199,303,020 (98.8) & $0.99(0.80-1.24)$ & 0.9580 & $0.86(0.67-1.10)$ & 0.2296 \\
\hline South & $4,328,261(1.2)$ & $362,166,243$ (98.8) & Referent & - & Referent & - \\
\hline \multicolumn{7}{|l|}{ MSA } \\
\hline Non-MSA & 1,709,090 (1.4) & 119,137,057 (98.6) & $1.18(0.93-1.52)$ & 0.1636 & $1.01(0.77-1.32)$ & 0.9462 \\
\hline MSA & $10,060,607(1.2)$ & $833,724,642$ (98.8) & Referent & - & Referent & - \\
\hline \multicolumn{7}{|l|}{ Insurance Status } \\
\hline Other & 407,967 (0.4) & $98,328,703$ (99.6) & $0.43(0.32-0.58)$ & $<0.0001$ & $0.51(0.36-0.72)$ & 0.0001 \\
\hline Medicaid & $407,549(0.5)$ & $87,966,848(99.5)$ & $0.48(0.35-0.66)$ & $<0.0001$ & $0.56(0.39-0.80)$ & 0.0014 \\
\hline Medicare & $6,193,910(2.2)$ & $274,708,378$ (97.8) & $2.35(2.07-2.68)$ & $<0.0001$ & $0.93(0.74-1.15)$ & 0.4846 \\
\hline Private Insurance & 4,382,477 (0.9) & $457,347,665$ (99.1) & Referent & - & Referent & - \\
\hline \multicolumn{7}{|l|}{ Setting Type } \\
\hline Hospital Emergency & $353,199(0.4)$ & $94,359,329(99.6)$ & $0.28(0.23-0.33)$ & $<0.0001$ & $0.42(0.34-0.51)$ & $<0.0001$ \\
\hline Hospital Outpatient & $892,347(1.1)$ & $77,244,784(98.9)$ & $0.86(0.71-1.03)$ & 0.0994 & $1.05(0.88-1.26)$ & 0.5718 \\
\hline Physician Office & $10,524,151(1.3)$ & $781,257,587$ (98.7) & Referent & - & Referent & - \\
\hline \multicolumn{7}{|l|}{ Diuretic Use } \\
\hline Yes & $3,575,442(5.0)$ & $67,667,807(95.0)$ & $5.71(5.04-6.47)$ & $<0.0001$ & $4.00(3.37-4.75)$ & $<0.0001$ \\
\hline No & $8,194,254(0.9)$ & $885,193,893$ (99.1) & Referent & - & Referent & - \\
\hline
\end{tabular}

MSA Metropolitan Statistical Area

* Survey weighting and clusters accounted for reflecting unbiased, national annual estimates of visit occurrences for the portion of the population meeting the study inclusion/exclusion criteria

Note that per the model fitting criterion described in the methods section, no variables were excluded from the multivariable model

important variables, demonstrated that significant associations remained between visits with a gout diagnosis and age group ( $\geq 65$ and $45-64$ vs. $20-44$ ), sex, ethnicity, race group ('Other' vs. Caucasian as well as now African-American vs. Caucasian), insurance status (Other and Medicaid vs. private insurance, but no longer 
Medicare vs. private insurance), hospital emergency departments (vs. physician office visits) and diuretic use. However, the associations between visits with a gout diagnosis and region ${ }^{1}$ of the country and as well as gout visits and Medicare (vs. private insurance) did not remain significant following adjustment for the effect of the other covariates of interest in the model. A diagnosis of gout remained more likely for patient visits in 20092011 as compared to visits in 2007-2008 (OR 1.24, 95\% CI 1.02-1.52). Additionally, a diagnosis of gout remained more likely for visits with patients 65 and older and patients 45-64 as compare to visits for those 20-44 (OR 4. 94, 95\% CI 3.69-6.62 and OR 2.52, 95\% CI 1.97-3.21, respectively). Gout was less likely to be diagnosed at visits for females as compared to males (OR 0.20, 95\% CI $0.18-0.24)$ and Hispanic/Latino patients as compared to Non-Hispanic/Latino patients (OR 0.64, 95\% CI $0.48-0.86$ ), while gout was more likely to be diagnosed at visits for those on a diuretic as compared to those not on a diuretic (OR 4.00, 95\% CI 3.37-4.75). Gout was less likely to be diagnosed at patient visits with an insurance status of "Other" and Medicaid as compared to those with private insurance (OR 0.51, 95\% CI $0.36-0.72$ and OR 0.56, 95\% CI 0.39-0.80, respectively). Patients whose visit was recorded in an emergency department were less likely (OR 0.42, 95\% CI 0.34-0.51) to be diagnosed with gout as compared to those with a physician's office visit (Table 2). Second order interaction terms were investigated, found to contribute nothing significant to the understanding of the overall results and were excluded from the final reported model.

Finally, plots of the percentage of patient visits with gout by year for medication class and individual drug showing the most common gout treatments can be seen in Figs. 1 and 2. The percent of gout visits in each drug class appears similar across the years studied. Antigout (80-86\%) and antihyperuricemic (67-75\%) medications were consistently the most common treatment while NSAIDs (14-20\%) and steroids (6-12\%) were a distant third and fourth, respectively.

Among individual medications, allopurinol (67-74\%) is clearly the most common, three to four times as likely as the next closest (colchicine). Febuxostat, probenecid and colchicine-probenecid were infrequently prescribed (all less than 5\%). Allopurinol and colchicine dominate the market, their use appears consistent across the years studied, while probenecid's use has decreased over time. Uptake does appear to be slow for febuxostat, the newest gout medication, as two years after entering the market, the percentage of patients with gout taking the drug remains around $3 \%$.

\section{Discussion}

The proportion of patient visits with a diagnosis of gout increased between 2007 and 2011, and this increase was significant. It is hypothesized that gout visits are on the rise due to increases in obesity, hypertension, and purine-rich diets $[1,2]$. Obesity increases the production of serum urate (sUA) levels and also decreases urate excretion while weight reduction has been associated with uric acid level declination [16]. Along with risk factors, there are many disease associations with gout including metabolic syndrome, hypertension, and cardiovascular disease [16]. Metabolic syndrome has been strongly associated with gout; $60 \%$ of US population with gout also has metabolic syndrome, a prevalence three times higher in those with gout [17]. Metabolic syndrome has likely

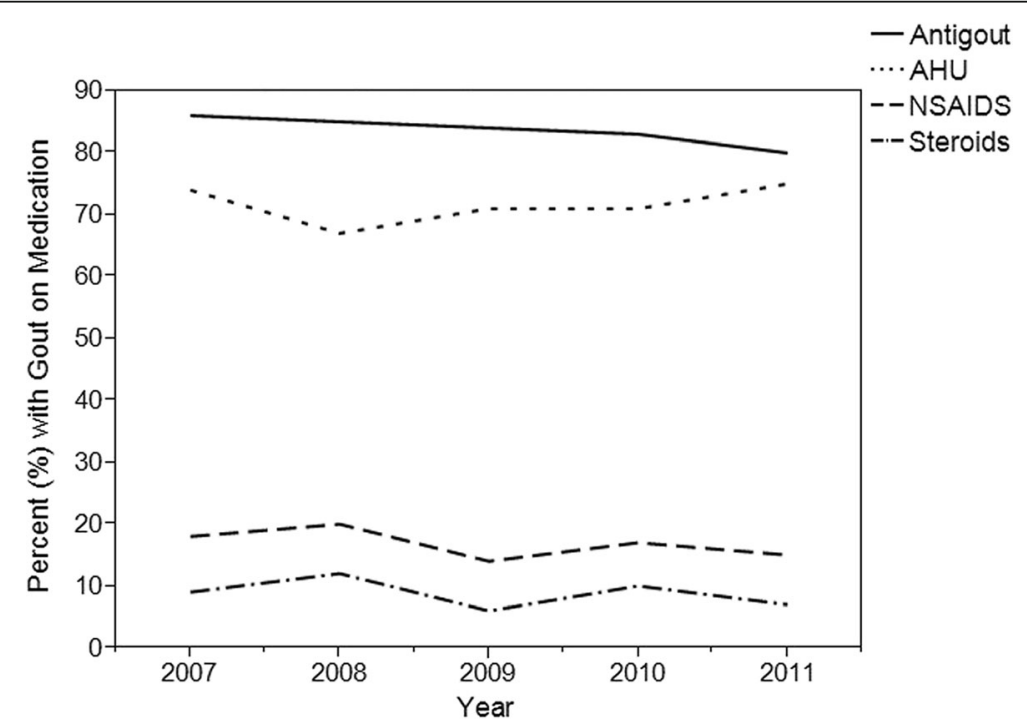

Fig. 1 Gout Prescription Trends by Drug Class, 2007-2011 


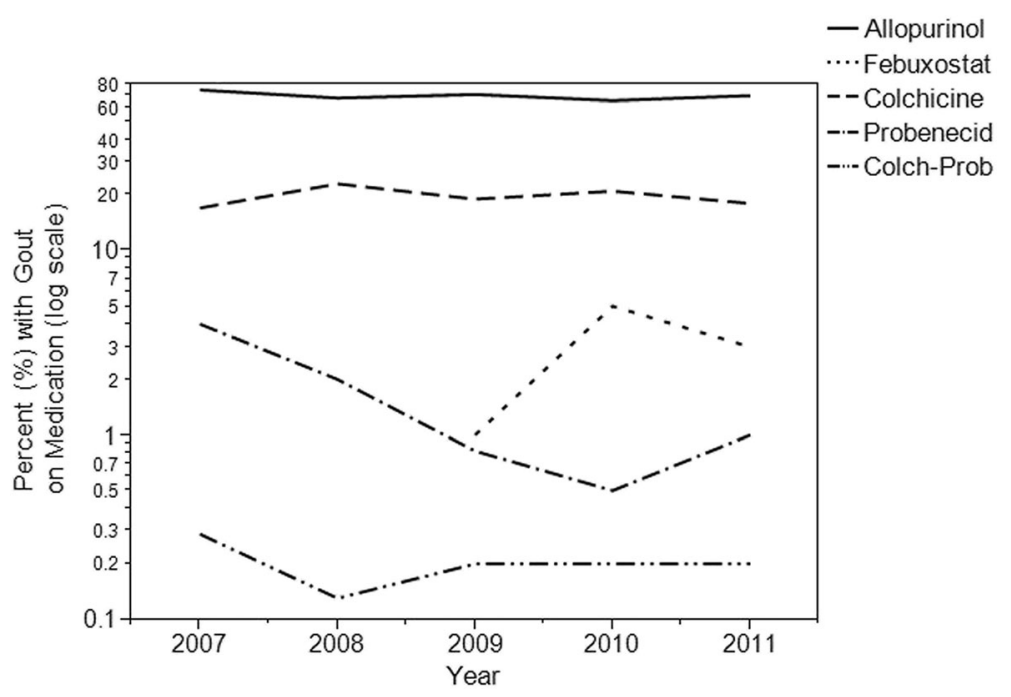

Fig. 2 Gout Prescription Trends by Individual Drug, 2007-2011

increased over the study years, helping explain the rise in gout diagnoses [18].

The number of gout visits in this study was not as high as noted in a similar prior study, [8] which could be attributed to several different factors. Although not specified in their methods, the Krishnan and Chen study appears to have used aggregated estimates for the years and databases studied, rather than the average annual estimates used in this study. Additionally, Krishnan and Chen only utilized NAMCS and NHAMCS-OPD, whereas this study also utilized NHAMCS-ED. Despite approximately 95 million visits attributed to the NHAMCS-ED, visits for gout in the ED were less likely. This likely increased the total number of overall visits without adding a commensurate number of gout-specific visits to the numerator. Further, while only $31 \%$ of the study population was aged 65 or older, $61 \%$ was female and the prevalence of gout is known to both increase with age and be more prevalent in males [3].

Another prior study with higher gout estimates by Zhu, et al. was based on participant reported data from the National Health and Nutrition Examination Survey (NHANES), which lends itself to estimating true prevalence [1]. This study is based on provider reported ambulatory, outpatient and emergency visits, therefore limiting the ability to estimate the true prevalence of gout. This database distinction helps explain this difference in gout estimates. It is worth noting that NHANES as well as the data sources used for this study are all population-based surveys.

The study results are consistent with several international epidemiology studies which examined the prevalence of gout [19-23]. The prevalence of gout has increased significantly in the United Kingdom (UK) over the years of 1997 through 2012 according to a study which utilized the Clinical Practice Datalink [19]. Another study which looked at gout prevalence in the UK and Germany from 2000 to 2005 with the IMS Disease Analyzer found a prevalence of 1.4\% [20]. A study of the Canadian province of British Columbia from 2000 to 2012 using PopulationDataBC found a prevalence of 3. $8 \%$ in 2012, and there was a noted increase over the study period [21]. A Swedish study examined gout trends from 2002 to 2012 and found a prevalence of 1 . $8 \%$ in 2012 as well as an increase over the study period. [22] A study in Taiwan utilizing the National Health Insurance Research Database found a higher prevalence rate of $6.24 \%$ over the study period of 2005 to 2010 [23]. With the exception of the Taiwan study [23], all studies were consistent with this study's findings with regards to gout prevalence and increasing prevalence over the years.

This study demonstrated an association between age, sex, race group and gout visits, with an increased proportion among older age groups ( $\geq 45$ years of age), males, African American and 'Other' race groups. These finding are consistent with previous studies $[1,3,8,10]$ showing that the risk of developing gout is age-related, $[1,8]$ and that estrogen is protective in premenopausal women due to its uricosuric effect [10]. 'Other' race within the databases consists of Asian, Native Hawaiian or other Pacific Islander, American Indian or Alaska Native, or more than one race reported. A higher prevalence of gout is well known in Asians and Pacific Islanders, as well as African Americans with genetics playing a role due to hyperuricemia-associated DNA sequence variations $[24,25]$. However, diet and the presence of co-morbidities cannot be ruled out. 
Hispanic/Latino individuals were found to be less likely to have a visit with gout than Non-Hispanic/Latinos. One possible explanation is related to diet. A previous study showed that Non-Hispanic/Latinos consume more red meat and seafood when compared to Hispanic/Latinos. [26] Diets rich in red meat and seafood are widely known to be associated with the production of uric acid [2]. Given that Hispanic/Latino diets are typically more heavily based on grains and beans along with fresh fruits and vegetables, Hispanic/ Latinos may produce less uric acid resulting in a lower incidence of gout [27].

Patient visits with Medicaid and 'Other' insurance were less likely to have a diagnosis of gout. Despite the lack of statistically significant interaction effects in the multivariable model, this could be attributed to the role of age with the risk of developing gout $[1,8]$. 'Other' insurance consisted of worker's compensation, self-pay, no charge/charity, and other, while Medicaid also included the Children's Health Insurance Program. Patients with 'Other' insurance or Medicaid are less likely to be older, the age group at the highest risk for gout.

Individuals were significantly less likely to have a visit with a diagnosis of gout in a hospital emergency setting than they were in a physician's office. Patients are more likely to visit a provider's office for routine check-ups and for chronic conditions like gout. While individuals may visit a hospital for an initial or particularly severe attack of gout, they are presumably more likely to visit their provider when simply attempting to help keep their gout under control. Furthermore, patients with gout are much more likely to visit their provider if they are being prescribed gout prophylaxis medication.

A study by Garg, et al. looked at gout-related health care utilization in US emergency departments utilizing the National Emergency Department Sample (NEDS) from 2006 to 2008 [28]. The Garg study found approximately $0.7 \%$ of ED visits to be gout-related, slightly higher than $0.4 \%$ found in this study [28]. A similar study by Jinno, et al. also utilized NEDS and examined gout ED visits from 2006 to 2012 [29]. This study found $0.19 \%$ of visits with a primary diagnosis of gout [29]. Although not exactly comparable to this study, which includes non-ED databases in addition to the NHAMCS-ED, similar findings with both of these studies include gout-related ED visits being more likely with men, and increasing age; and less likely with different insurance types [28, 29].

Diuretic use was four times more likely to be associated with a gout visit. Previous research has shown that individuals who have high blood pressure and are also taking a diuretic have an increased risk for acquiring gout [6]. The diuretics' mechanism of action is thought to contribute to gout, increasing uric acid reabsorption [30].

The graph of gout medication class by year showed consistency in use among the drug classes over the years. Antigout and antihyperuricemic medication classes remained the two most commonly prescribed treatments, while NSAIDs and steroids were used less. It is worth noting that due to drug class coding within the databases some medications could have been coded in both the antigout and antihyperuricemic class (i.e., allopurinol and febuxostat) since drugs may be coded in as many as four different medication classes. This might explain why the antigout percentage is greater than the antihyperuricemics. However, the findings in this study are consistent with the prior NAMCS and NHAMCSOPD study which looked at gout treatment trends up through 2009 [8]. These treatment trends can also be explained by typical prescribing patterns for a gouty attack versus prophylaxis treatment to prevent gout flare. NSAIDs and steroids are typically only used for gouty attacks and patients are treated prophylactically after an initial gout attack to prevent future attacks [2, 31]. In addition, the risk of side effects with NSAIDs such as gastrointestinal bleeds, renal failure, and hypertension likely impacted their use in treatment, especially in the case when chronic treatment is warranted [32, 33].

As evident from the figure showing the percentage of visits by year for individual gout drugs, allopurinol continues to be the most prescribed treatment with colchicine second, a finding also consistent with Krishnan and Chen [8]. Allopurinol dominated the market as the only medication to reduce uric acid synthesis until the introduction of febuxostat in 2009 [2, 4, 11]. As expected, the percentage of visits with febuxostat increased following its approval. Despite this, allopurinol and colchicine use changed little from 2009 through 2011, evidence that febuxostat introduction had minimal impact on the treatment trends for the study years. Probenecid use has declined over the years which can be explained by its potential for drugdrug interactions as well as less favorable side effect profile, including risk of urolithiasis [2, 11, 34].

The previously mentioned international studies showed similar treatment trends. Allopurinol was prescribed for most patients in UK and Germany at 89 and 93\% respectively; while colchicine use was only around $15-16 \%$ for both [20]. Probenecid use was minimal $(<1 \%)$, but NSAIDs were utilized $80-90 \%$ for prophylaxis. [20]. Allopurinol was also most commonly prescribed in British Columbia, Canada, with less than $1 \%$ use of febuxostat and probenecid [21]. Colchicine and steroid use increased in British Columbia over the study period, while NSAID use declined by 31\% [21]. A study in Australia in 2005 found allopurinol to comprise $98.4 \%$ of all urate lowering therapy with probenecid at $<1 \%$ [35]. There was a common theme from these studies of the overall underutilization of uratelowering treatment for gout [19-23, 35].

The study is not without limitations. The observational, cross-sectional nature of the study design limited the 
authors to statements of association between visits with gout diagnosis and the factors of interest. No claims of causality can be made. Furthermore, the cross-sectional nature of the data sources used did not allow for repeated measurements on patients over time. Several variables of interest, including alcoholism, Parkinson's disease, depression, hypertension, weight status, tobacco use, and losartan use had to be excluded from all analyses due to missing data and/or reliability issues. This is particularly unfortunate for variables such as hypertension and weight status, both known to be significantly associated with gout. All of the databases utilized are limited to three diagnoses. The NAMCS and NHAMCS-OPD include a data field to collect other specific disease states (includes hypertension, diabetes, and depression), but NHAMCS-ED does not and only collects the diabetes variable of interest in their other specific disease field. This likely contributed to the missing data for such highly prevalent conditions like hypertension and diabetes. The NAMCS and NHAMCS databases do not include federal offices or hospitals, including Veterans Affairs facilities where gout can be prevalent. In addition, the databases do not provide a true prevalence of gout, but rather a surrogate via visits for gout based on diagnostic codes from the three recorded diagnoses and gout medications prescribed at the visits. It is not uncommon for epidemiological studies to rely on diagnostic codes for estimating prevalence. Those studies which have relied on such codes have shown good accuracy. In addition, although gout medications were also used to identify gout visits, there is a chance that medications like colchicine and probenecid were used for conditions other than gout. However, such alternative uses are rare. Study strengths include the use of nationally representative, population-based surveys which allow for generalizing findings to the portion of the US population that is commensurate with the study population. Further, the databases are provider reported data which allows for more reliability of results as compared to patient reported data. This is the first study known to the authors to investigate febuxostat in the treatment of gout since its approval in 2009 [4].

\section{Conclusion}

This study found that the proportion of visits with a diagnosis of gout continues to increase, although the overall percentage of gout remains low in the US population. Individuals who are male, aged 45-64 or 65 and older, non-Hispanic/Latino, African American or of 'Other' race, use private insurance, present to a physician's office, or use a diuretic are more likely to have a visit with a diagnosis of gout. Treatment trends over the study years by medication class and individual gout medications have remained consistent, with the introduction of febuxostat having little impact for the study years through 2011.
Poster presentations 2016 Wiggins Academic Symposium, Campbell University, Buies Creek, NC.

2017 Interprofessional Health Sciences Research Symposium, Campbell University, Buies Creek, NC.

\section{Endnotes}

${ }^{1}$ The NAMCS and NHAMCS surveys define the four regions that comprise this variable as follows:Northeast: Connecticut, Maine, Massachusetts, New Hampshire, New Jersey, New York, Pennsylvania, Rhode Island, Vermont; Midwest: Illinois, Indiana, Iowa, Kansas, Michigan, Minnesota, Missouri,Nebraska, North Dakota, Ohio, South Dakota, Wisconsin; South: Alabama, Arkansas, Delaware, District of Columbia, Florida, Georgia, Kentucky, Louisiana, Maryland, Mississippi, North Carolina,Oklahoma, South Carolina, Tennessee, Texas, Virginia, West Virginia; West: Arizona, California, Colorado, Idaho, Montana, Nevada, New Mexico, Oregon, Utah, Washington, Wyoming, Alaska, Hawaii

\section{Abbreviations}

Cls: Confidence intervals; MSA: Metropolitan statistical area; NAMCS: National Ambulatory Medical Care Survey; NCHS: National Center for Health Statistics; NEDS: National Emergency Department Sample; NHAMCS-OPD/NHAMCSED: National Hospital Ambulatory Medical Care Survey Outpatient Department \& Emergency Department; NHANES: National Health and Nutrition Examination Survey; ORs: Odds ratios; sUA: Serum urate

\section{Availability of data and materials}

The datasets used and/or analysed during the current study are available from the corresponding author on reasonable request. Note that the survey data from which the analysis dataset was constructed can be found at the CDC website https://www.cdc.gov/nchs/ahcd/index.htm.

\section{Authors' contributions}

KEC, KDC, DR, and MH wrote the Background, Discussion, Results, and Conclusions sections of the manuscript. MJ conducted all analyses and wrote the Methods section of the manuscript. All authors read and approved the final manuscript.

Ethics approval and consent to participate

The study was submitted to the Campbell University Institutional Review Board and received an exemption due to the data sources used being publicly available and de-identified. As such, since this research was based solely on the analysis of previously collected, de-identified data, it complies with the Helsinki Declaration.

Competing interests

The authors declare that they have no competing interests.

\section{Publisher's Note}

Springer Nature remains neutral with regard to jurisdictional claims in published maps and institutional affiliations.

Received: 8 January 2018 Accepted: 24 April 2018

Published online: 30 May 2018

\section{References}

1. Zhu Y, Pandya BJ, Choi HK. Prevalence of gout and hyperuricemia in the US general population. Arthritis Rheum. 2011;63:3136-41.

2. Rymal E, Rizzolo D. Gout a comprehensive review. JAAPA. 2014;27:26-31.

3. Sunkureddi PS, Nguyen-Oghalai TU, Karnath BM. Clinical signs of gout. Hospital Physician. 2006;42:39-42,47. 
4. U.S. Food and Drug Administration. FDA Approved Drug Products. http:// www.accessdata.fda.gov/scripts/cder/drugsatfda/index.cfm?fuseaction= Search.DrugDetails. Accessed 10 Aug 2016.

5. Choi HK, Atkinson K, Karlson EW, Curhan G. Obesity, weight change, hypertension, diuretic use, and risk of gout in men: the health professionals follow-up study. Arch Intern Med. 2005;165:742-8.

6. Primatesta P, Plana E, Rothenbacher D. Gout treatment and comorbidities: a retrospective cohort study in a large US managed care population. BMC Musculoskelet Disord. 2011;12:103.

7. Juraschek SP, Miller ER, Gelber AC. Body mass index, obesity, and prevalent gout in the United States in 1988-1994 and 2007-2010. Arthritis Care Res. 2013;65:127-32.

8. Krishnan E, Chen L. Trends in physician diagnosed gout and gout therapies in the US: results from the national ambulatory health care surveys 1993 to 2009. Arthritis Res Ther. 2013;15:R181.

9. Lee SJ, Hirsch JD, Terkeltaub R, Khanna D, Singh JA, Sarkin A, et al. Perceptions of disease and health-related quality of life among patients with gout. Rheumatology (Oxford). 2009;48:582-6.

10. Bhole V, de Vera M, Rahman M, Krishnan E, Choi H. Epidemiology of gout in women: fifty-two-year followup of a prospective cohort. Arthritis Rheum. 2010;62:1069-76

11. Baker JF, Schumacher HR. Update on gout and hyperuricemia. Int J Clin Pract. 2010;64:371-7.

12. McCaig LF, Burt CW. Understanding and interpreting the National Hospital Ambulatory Medical Care survey: key questions and answers. Ann Emerg Med. 2012;60:716-21.

13. Sample text for describing NHAMCS in a research article. https://www.cdc. gov/nchs/data/ahcd/Sample_Text_for_Describing_NHAMCS_in_Research_ Article.pdf. Accessed 1 Mar 2018.

14. National Center for Health Statistics. Scope and sample design. http://www. cdc.gov/nchs/ahcd/ahcd_scope.htm\#namcs_scope. Accessed 1 Mar 2018.

15. SAS Institute Inc. SAS/STAT 9.3 User's Guide. Cary: SAS Institute Inc; 2011.

16. Saag KG, Choi H. Epidemiology, risk factors, and lifestyle modifications for gout. Arthritis Res Ther. 2006;8(1):S2

17. Choi HK, Ford ES, Li C, Curhan G. Prevalence of the metabolic syndrome in patients with gout: the third National Health and nutrition examination survey. Arthritis Rheum. 2007;57:109-15.

18. Aguilar M, Bhuket T, Torres S, Liu B, Wong RJ. Prevalence of the metabolic syndrome in the United States, 2003-2012. JAMA. 2015;313:1973-4.

19. Kuo CF, Grainge MJ, Mallen C, Zhang W, Doherty M. Rising burden of gout in the UK but continuing suboptimal management: a nationwide population study. Ann Rheum Dis. 2015;74:661-7.

20. Annemans L, Spaepen E, Gaskin M, Bonnemaire M, Malier V, Gilbert T, et al. Gout in the UK and Germany: prevalence, comorbidities and management in general practice 2000-2005. Ann Rheum Dis. 2008;67:960-6.

21. Rai SK, Avina-Zubieta JA, McCormick N, De Vera MA, Shojania K, Sayre EC, et al. The rising prevalence and incidence of gout in British Columbia, Canada: population-based trends from 2000 to 2012. Semin Arthritis Rheum. 2017; 46:451-6.

22. Dehlin M, Drivelegka P, Sigurdardottir V, Svard A, Jacobsson LTH. Incidence and prevalence of gout in western Sweden. Arthritis Res Ther. 2016;18:164

23. Kuo CF, Grainge MJ, See LC, Yu KH, Luo SF, Zhang W, et al. Epidemiology and management of gout in Taiwan: a nationwide population study. Arthritis Res Ther. 2015:17:13.

24. Reginato AM, Mount DB, Yang I, Choi HK. The genetics of hyperuricaemia and gout. Nat Rev Rheumatol. 2012:8:610-21.

25. Yang Q, Köttgen A, Dehghan A, Smith AV, Glazer NL, Chen MH, et al. Multiple genetic loci influence serum urate levels and their relationship with gout and cardiovascular disease risk factors. Circ Cardiovasc Genet. 2010;3:523-30.

26. Winkleby MA, Albright CL, Howard-Pitney AB, Lin J, Fortmann SP. Hispanic/ white differences in dietary fat intake among low educated adults and children. Prev Med. 1994;23:465-73.

27. Diet.com. Hispanic and latino diet. http://www.diet.com/g/hispanic-andlatino-diet. Accessed 1 Mar 2018.

28. Garg R, Sayles HR, Yu F, Michaud K, Singh J, Saag KG, et al. Gout-related health care utilization in US emergency departments, 2006 through 2008. Arthritis Care Res. 2013;65:571-7.

29. Jinno S, Hasegawa K, Neogi T, Goho T, Dubreuil M. Trends in emergency department visits and charges in the United States between 2006 and 2012 J Rheumatol. 2016:43:1589-92.
30. Pascual E, Perdiguero M. Gout, diuretics and the kidney. Ann Rheum Dis. 2006;65:981-2.

31. Rees F, Hui M, Doherty M. Optimizing current treatment of gout. Nat Rev Rheumatol. 2014;10:271-83.

32. Moon KW, Kim J, Kim JH, Song R, Lee EY, Song YW, et al. Risk factors for acute kidney injury by non-steroidal anti-inflammatory drugs in patients with hyperuricemia. Rheumatology. 2011;50:2278-82.

33. Madhok R, Wu O, McKellar G, Singh G. Non-steroidal anti-inflammatory drugs-changes in prescribing may be warranted. Rheumatology. 2006;45: 1458-60.

34. Reinders MK, van Roon EN, Jansen TL, Deising J, Griep EN, Hoekstra M, et al. Efficacy and tolerability of urate-lowering drugs in gout: a randomized controlled trial of benzbromarone versus probenecid after failure of allopurinol. Ann Rheum Dis. 2009;68:51-6.

35. Chung Y, Lu CY, Graham GG, Mant A, Day RO. Utilization of allopurinol in the Australian community. Int Med J. 2008;38:388-95.

\section{Ready to submit your research? Choose BMC and benefit from:}

- fast, convenient online submission

- thorough peer review by experienced researchers in your field

- rapid publication on acceptance

- support for research data, including large and complex data types

- gold Open Access which fosters wider collaboration and increased citations

- maximum visibility for your research: over $100 \mathrm{M}$ website views per year

At BMC, research is always in progress.

Learn more biomedcentral.com/submissions 